\title{
Mild Isomerative Opening of Tetrahydrofuranyl Subunits in Steroids Using TFAT (trifluoroacetyl trifluoromethanesulfonate): Application to Synthesis of C17-OH Rockogenin Acetate
}

\author{
Jong Seok Lee, 'Byungsook Kim, Junho Shin, Yeon-Ju Lee, Hee Jae Shin, and Hyi-Seung Lee \\ Marine Natural Products Laboratory, Korea Ocean Research and Development Institute, Ansan 426-744, Korea \\ *E-mail: jslee@kordi.re.kr \\ Received October 20, 2011, Accepted November 2, 2011
}

\begin{abstract}
A novel and efficient tetrahydrofuranyl ring opening method was developed using the highly reactive TFAT reagent in the presence of an acid scavenger, 2,6-di-tert-butyl-4-methylpyridine. Various acid sensitive groups are compatible with the reaction condition, making it generally applicable to many tetrahydrofuranyl steroids. Moreover, it is a synthetic equivalent of 'Marker degradation' affording an efficient synthesis of $\mathrm{C} 17-\mathrm{OH}$ rockogenin acetate.
\end{abstract}

Key Words : Steroid, TFAT (trifluoroacetyl trifluoromethanesulfonate), Ring opening, Dihydroxylation, Alkoxyl radical cyclization

\section{Introduction}

In modern organic synthesis, the ether is one of the most frequently found features. It is commonly used as a protecting group for alcohols. ${ }^{1}$ At the same time, its cyclic forms such as tetrahydrofurans and pyrans are found as core structures in many biologically active natural products. During the last few decades, many methods have been developed to manipulate the ethers, which are mainly focused on their introduction and cleavage. ${ }^{1}$ Cleavage of cyclic ethers is an essential process in the syntheses of many natural products since it enables easy introduction of additional functionalities. ${ }^{2}$

Over many years, steroid sapogenins have been standing as steadfast starting materials for the synthesis of steroidal natural products. Thus, various reactions were developed and applied to chemically modify the steroid sapogenins. ${ }^{2 \mathrm{~g}, 2 \mathrm{~h}, 3-5}$

Since the historic report in 1938, 'Marker degradation' has been generally used for the synthesis of steroidal natural products including sex hormones such as progesterone, testosterone, estradiol and other steroid analogs. ${ }^{4}$ Especially, it is the essential early process for introduction of C17- $\alpha-\mathrm{OH}$, which was successfully applied to the previous first generation synthesis of cephalostatin 1 and 7 (Scheme 1)..$^{5}$

But application of 'Marker degradation' in the synthesis of cephalostatins includes excision of the entire F-ring in the steroid sapogenin and subsequent reintroduction of the same atoms, which is not attractive on the strategic level (Scheme 1). To overcome this shortcoming, it is required to develop a novel synthetic tool retaining all carbon atoms of the starting steroid sapogenin and employ specific oxidation reactions

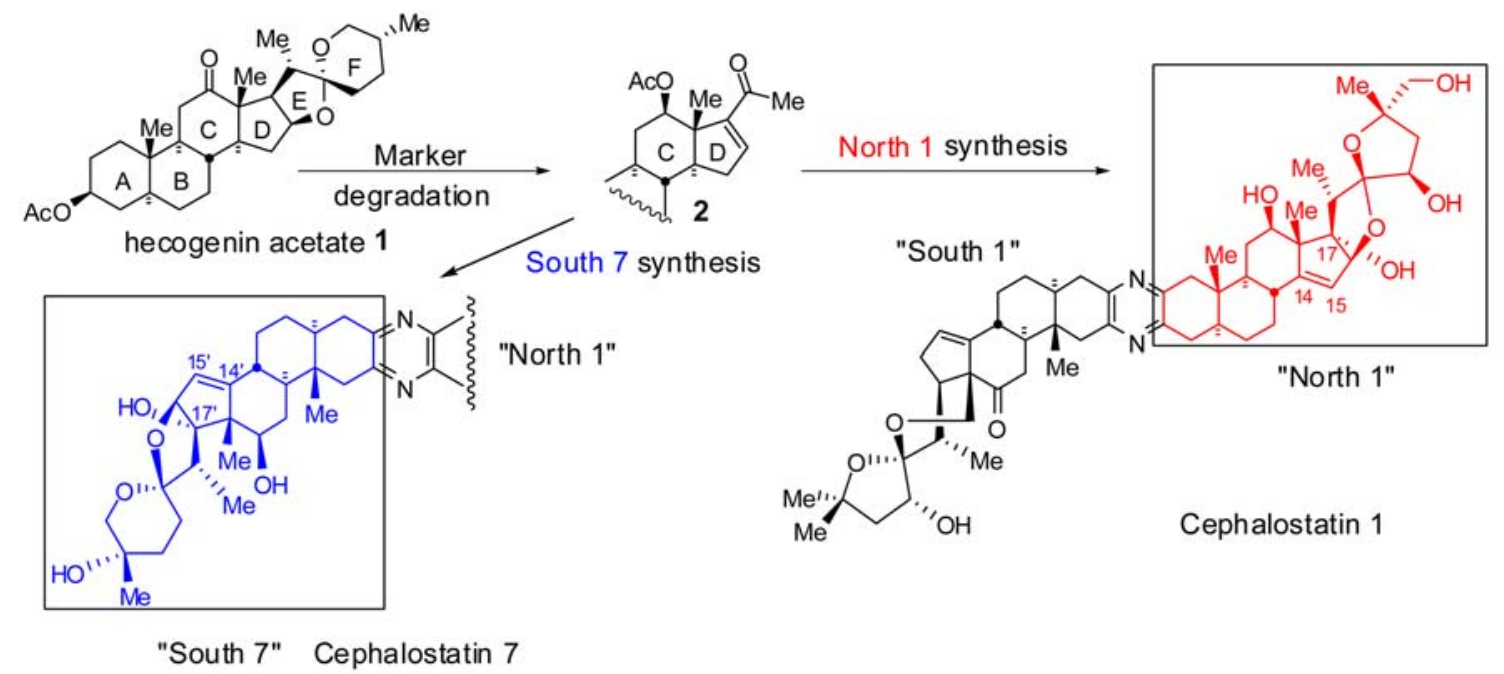

Scheme 1. The first generation synthesis of cephalostatins. 
for introduction of the desired functional groups to the specific positions.

\section{Experimental Section}

All reactions were carried out under nitrogen unless otherwise indicated. Anhydrous methylene chloride $\left(\mathrm{CH}_{2} \mathrm{Cl}_{2}\right)$, diethyl ether, and tetrahydrofuran (THF) were directly used from commercial source. Pyridine, cyclohexane, and triethylamine were used without further purification. All work-up, wash, and chromatographic solvents were distilled. Sodium sulfate $\left(\mathrm{Na}_{2} \mathrm{SO}_{4}\right)$ was anhydrous.

Thin layer chromatography (TLC) was used to monitor the progress of reactions by co-spotting with the starting materials. $p$-Anisaldehyde $(1350 \mathrm{~mL}$ absolute ethanol, 50 $\mathrm{mL}$ concentrated $\mathrm{H}_{2} \mathrm{SO}_{4}, 37 \mathrm{~mL} p$-anisaldehyde) was utilized as a common TLC visualizing solution.

Flash chromatographic purifications were performed using silica gel (230-400 mesh). ${ }^{1} \mathrm{H},{ }^{13} \mathrm{C}$, and ${ }^{19} \mathrm{~F}$ NMR spectra were recorded as solutions in chloroform- $\mathrm{d}_{1}$ and are described in parts per million (ppm) from the residual chloroform (7.24 ppm and $77.23 \mathrm{ppm}$ ).

Peak multiplicates in ${ }^{1} \mathrm{H}$ NMR spectra are abbreviated as $\mathrm{s}$ (singlet), d (doublet), t (triplet), $m$ (multiplet), dd (doublet of doublet), q (quartet), and dt (doublet of triplet).

General Procedure for the Preparation of Compounds $4,5,7,9,11$, and 13 . To a solution of a tetrahydrofuranyl steroid (compound $3, \mathbf{6}, \mathbf{8}, \mathbf{1 0}, \mathbf{1 2}, \mathbf{1 4}$ ) and 2,6-di-tert-butyl4-methylpyridine (indicated in the main text) in anhydrous
$\mathrm{CH}_{2} \mathrm{Cl}_{2}$ (0.1-0.15 M) was added freshly prepared TFAT (3-5 equiv. as indicated in Table 1 of the main text) at $-35^{\circ} \mathrm{C}$. The reaction was stirred at $-35^{\circ} \mathrm{C}$ until the starting material disappeared on TLC (normally $40 \mathrm{~min}$. to $2 \mathrm{~h}$ ), then quenched with sat. $\mathrm{NaHCO}_{3}$. The reaction was extracted with EtOAc three times. The extract was washed with brine, dried over anhydrous $\mathrm{Na}_{2} \mathrm{SO}_{4}$, filtered, and concentrated in vacuo. The crude product was purified by flash column chromatography (EtOAc/n-Hexane $1: 10$ to $1: 6$ ) to give the corresponding product (compound 4, 5, 7, 9, 11, and 13).

Trifluoroacetate 4: Terminal olefin 3 (96 mg, $0.17 \mathrm{mmol}$ ) was used and the reaction was carried out with 3 equiv. of TFAT $(0.52 \mathrm{mmol}, 0.08 \mathrm{~mL})$ alone to provide compound 4 (79 mg, $0.12 \mathrm{mmol}, 70 \%$, white floppy solid): ${ }^{1} \mathrm{H}$ NMR (300 $\left.\mathrm{MHz}, \mathrm{CDCl}_{3}\right) \delta 8.03(\mathrm{~d}, J=7.2 \mathrm{~Hz}, 2 \mathrm{H}), 7.55(\mathrm{t}, J=7.3 \mathrm{~Hz}$, $1 \mathrm{H}), 7.41-7.46(\mathrm{~m}, 2 \mathrm{H}), 6.15(\mathrm{~d}, J=1.8 \mathrm{~Hz}, 1 \mathrm{H}), 5.88$ (s, $1 \mathrm{H}), 5.06(\mathrm{q}, J=6.3 \mathrm{~Hz}, 1 \mathrm{H}), 4.96(\mathrm{t}, J=7.10 \mathrm{~Hz}, 1 \mathrm{H}), 4.61-$ $4.70(\mathrm{~m}, 1 \mathrm{H}), 4.40(\mathrm{dd}, J=11.4,4.2 \mathrm{~Hz}, 1 \mathrm{H}), 2.86$ (quintet, $J$ $=7.0 \mathrm{~Hz}, 1 \mathrm{H}), 2.27-2.30(\mathrm{~m}, 2 \mathrm{H}), 2.17(\mathrm{t}, J=10.6 \mathrm{~Hz}, 1 \mathrm{H})$, $1.97(\mathrm{~s}, 3 \mathrm{H}), 1.60(\mathrm{~s}, 3 \mathrm{H}), 1.47(\mathrm{~s}, 3 \mathrm{H}), 1.23(\mathrm{~s}, 3 \mathrm{H}), 0.89(\mathrm{~s}$, $3 \mathrm{H}), 0.85(\mathrm{~d}, J=6.9 \mathrm{~Hz}, 3 \mathrm{H}) ;{ }^{13} \mathrm{C} \mathrm{NMR}\left(75 \mathrm{MHz}, \mathrm{CDCl}_{3}\right) \delta$ $170.4,165.4,156.9,156.5\left(\mathrm{q},{ }^{2} J_{\mathrm{C}-\mathrm{F}}=41.6 \mathrm{~Hz}, \mathrm{C}=\mathrm{O}\right), 155.7$, $135.6,133.0,130.3,129.3,128.4,125.9,120.8,118.4$ (q, $\left.{ }^{1} J_{\mathrm{C}-\mathrm{F}}=288.4 \mathrm{~Hz}, \mathrm{CF}_{3}\right), 117.5,82.1,79.2,73.2,56.8,53.1$, 44.2, 36.9, 35.7, 35.4, 34.6, 33.7, 29.6, 29.0, 28.0, 27.2, 27.1, 25.6, 21.2, 18.5, 17.5, 13.6, 12.0; ${ }^{19} \mathrm{~F}$ NMR (282 MHz, $\left.\mathrm{CDCl}_{3}\right) \delta-75.1$; HRMS (ESI) for $\mathrm{C}_{38} \mathrm{H}_{47} \mathrm{~F}_{3} \mathrm{O}_{6} \mathrm{Na}[\mathrm{M}+\mathrm{Na}]^{+}$ calcd. 679.3223 , found 679.3233 .

Trifluoroacetate 7: TBDPS ether 6 (166 mg, $0.18 \mathrm{mmol})$

Table 1. Results of the TFAT-promoted E-ring opening of steroids

Entry $^{a}$

${ }^{a}$ All the reactions were performed at $-35^{\circ} \mathrm{C} .{ }^{b}$ Freshly prepared TFAT was used. ${ }^{c}$,6-di-tert-butyl-4-methylpyridine. ${ }^{d}$ Separated yields. 
was used and the reaction was carried out with 3 equiv. of TFAT $(0.54 \mathrm{mmol}, 0.08 \mathrm{~mL})$ and 1.20 equiv. of 2,6-di-tertbutyl-4-methylpyridine $(0.21 \mathrm{mmol}, 44.0 \mathrm{mg})$ to provide compound 7 (167 mg, $0.16 \mathrm{mmol}, 91 \%$, white floppy solid): ${ }^{1} \mathrm{H}$ NMR (300 MHz, $\left.\mathrm{CDCl}_{3}\right) \delta 8.02(\mathrm{~d}, J=7.3 \mathrm{~Hz}, 2 \mathrm{H}), 7.58$ $(\mathrm{d}, J=7.0 \mathrm{~Hz}, 4 \mathrm{H}), 7.50(\mathrm{t}, J=7.2 \mathrm{~Hz}, 1 \mathrm{H}), 7.34-7.45(\mathrm{~m}$, $8 \mathrm{H}), 6.13(\mathrm{~d}, J=1.6 \mathrm{~Hz}, 1 \mathrm{H}), 5.89(\mathrm{~s}, 1 \mathrm{H}), 5.05-5.07(\mathrm{~m}$, $1 \mathrm{H}), 4.65-4.72(\mathrm{~m}, 1 \mathrm{H}), 4.40$ (dd, $J=11.2,4.1 \mathrm{~Hz}, 1 \mathrm{H}), 3.78$ (t, $J=11.0 \mathrm{~Hz}, 1 \mathrm{H}), 3.67$ (t, $J=10.0 \mathrm{~Hz}, 1 \mathrm{H}$ ), 2.86 (quintet, $J=6.9 \mathrm{~Hz}, 1 \mathrm{H}), 2.20($ br t $, J=10.1 \mathrm{~Hz}, 1 \mathrm{H}), 2.00(\mathrm{~s}, 3 \mathrm{H})$, $1.40(\mathrm{~s}, 3 \mathrm{H}), 1.24(\mathrm{~s}, 3 \mathrm{H}), 1.00(\mathrm{~s}, 9 \mathrm{H}), 0.92(\mathrm{~s}, 3 \mathrm{H}), 0.85(\mathrm{~d}$, $J=9.08 \mathrm{~Hz}, 3 \mathrm{H}) ;{ }^{13} \mathrm{C} \mathrm{NMR}\left(75 \mathrm{MHz}, \mathrm{CDCl}_{3}\right) \delta 170.6$, $165.5,156.6\left(\mathrm{q},{ }^{2} J_{\mathrm{C}-\mathrm{F}}=45.0 \mathrm{~Hz}, \mathrm{C}=\mathrm{O}\right), 156.2,156.0,155.8$ $\left(\mathrm{q},{ }^{2} J_{\mathrm{C}-\mathrm{F}}=45.0 \mathrm{~Hz}, \mathrm{C}=\mathrm{O}\right), 135.5,133.1,132.6,132.5,130.2$, $129.9,129.3,128.5,127.8,126.5,120.8,114.2\left(\mathrm{q},{ }^{1} J_{\mathrm{C}-\mathrm{F}}=\right.$ $277.8 \mathrm{~Hz}, \mathrm{C}=\mathrm{O}), 114.0\left(\mathrm{q},{ }^{1} J_{\mathrm{C}-\mathrm{F}}=277.8 \mathrm{~Hz}, \mathrm{C}=\mathrm{O}\right), 89.4$, $89.3,82.0,79.3,73.3,66.3,56.9,53.2,44.3,37.0,35.8$, $35.5,34.7,33.8,31.6,30.8,29.1,28.1,27.3,27.2,26.6$, $25.3,24.2,22.6,21.4,20.3,19.1,18.1,14.1,13.6,12.2 ;{ }^{19} \mathrm{~F}$ NMR (282 MHz, $\left.\mathrm{CDCl}_{3}\right) \delta-74.9,-75.4$; HRMS (ESI) for $\mathrm{C}_{56} \mathrm{H}_{66} \mathrm{~F}_{6} \mathrm{O}_{9} \mathrm{SiNa}[\mathrm{M}+\mathrm{Na}]^{+}$calcd. 1047.4278, found 1047.4280.

Trifluoroacetate 9:. Terminal olefin 8 (103 mg, 0.18 mmol) was used and the reaction was carried out with 3 equiv. of TFAT $(0.55 \mathrm{mmol}, 0.08 \mathrm{~mL})$ and 1.20 equiv. of 2,6di-tert-butyl-4-methylpyridine $(0.22 \mathrm{mmol}, 45 \mathrm{mg})$ to provide compound $9(73 \mathrm{mg}, 0.11 \mathrm{mmol}, 61 \%$, white floppy solid): ${ }^{1} \mathrm{H}$ NMR $\left(300 \mathrm{MHz}, \mathrm{CDCl}_{3}\right) \delta 8.04(\mathrm{~d}, J=7.2 \mathrm{~Hz}$, 2H), $7.56(\mathrm{t}, J=7.3 \mathrm{~Hz}, 1 \mathrm{H}), 7.43-7.48(\mathrm{~m}, 2 \mathrm{H}), 5.41(\mathrm{~s}$, $1 \mathrm{H}), 5.09-5.16(\mathrm{~m}, 2 \mathrm{H}), 4.63-4.70(\mathrm{~m}, 2 \mathrm{H}), 4.49(\mathrm{~s}, 1 \mathrm{H})$, 2.64 (quintet, $J=7.7 \mathrm{~Hz}, 1 \mathrm{H}), 1.99(\mathrm{~s}, 3 \mathrm{H}), 1.56(\mathrm{~s}, 3 \mathrm{H})$, 0.98 (s, 3H), 0.98 (s, 3H), $0.83(\mathrm{~s}, 3 \mathrm{H}), 0.82(\mathrm{~d}, J=7.0 \mathrm{~Hz}$, $3 \mathrm{H}) ;{ }^{13} \mathrm{C}$ NMR $\left(75 \mathrm{MHz}, \mathrm{CDCl}_{3}\right) \delta 170.6,165.7,157.0(\mathrm{q}$, $\left.{ }^{2} J_{\mathrm{C}-\mathrm{F}}=39.0 \mathrm{~Hz}, \mathrm{C}=\mathrm{O}\right), 154.2,144.1,133.1,130.4,129.4$, $128.5,123.5,118.6\left(\mathrm{q},{ }^{1} J_{\mathrm{C}-\mathrm{F}}=288.0 \mathrm{~Hz}, \mathrm{CF}_{3}\right), 110.5,81.1$, 78.7, 73.4, 54.2, 53.1, 50.5, 44.7, 36.4, 35.7, 35.6, 33.8, 33.1, 31.9, 31.2, 30.7, 29.4, 28.3, 27.4, 27.2, 22.3, 21.4, 18.2, 12.0; ${ }^{19} \mathrm{~F}$ NMR (282 $\left.\mathrm{MHz}, \mathrm{CDCl}_{3}\right) \delta-75.0$; HRMS (ESI) for $\mathrm{C}_{38} \mathrm{H}_{49} \mathrm{~F}_{3} \mathrm{O}_{6} \mathrm{Na}[\mathrm{M}+\mathrm{Na}]^{+}$calcd. 681.3379 , found 681.3375 .

Trifluoroacetate 11: TBDPS ether 10 (522 mg, $0.64 \mathrm{mmol})$ was used and the reaction was carried out with 5 equiv. of TFAT (3.19 mmol, $0.49 \mathrm{~mL})$ and 5 equiv. of 2,6-di-tertbutyl-4-methylpyridine $(3.19 \mathrm{mmol}, 655 \mathrm{mg})$ to provide compound 11 (396 mg, $0.43 \mathrm{mmol}, 68 \%$, white floppy solid): ${ }^{1} \mathrm{H} \mathrm{NMR}\left(500 \mathrm{MHz}, \mathrm{CDCl}_{3}\right) \delta 8.02(\mathrm{~d}, J=8.1 \mathrm{~Hz}$, 2H), $7.72(\mathrm{~d}, J=7.6 \mathrm{~Hz}, 1 \mathrm{H}), 7.63(\mathrm{t}, J=7.7 \mathrm{~Hz}, 4 \mathrm{H}), 7.33-$ $7.43(\mathrm{~m}, 9 \mathrm{H}), 5.40(\mathrm{~s}, 1 \mathrm{H}), 5.11-5.16(\mathrm{~m}, 2 \mathrm{H}), 4.66-4.71(\mathrm{~m}$, $1 \mathrm{H}), 3.30$ (t, $J=5.0 \mathrm{~Hz}, 1 \mathrm{H}), 3.30$ (q, $J=10.0 \mathrm{~Hz}, 1 \mathrm{H}), 2.64$ (quintet, $J=7.4 \mathrm{~Hz}, 1 \mathrm{H}), 2.05-2.15(\mathrm{~m}, 2 \mathrm{H}), 2.00(\mathrm{~d}, J=1.2$ $\mathrm{Hz}, 3 \mathrm{H}), 1.06$ (s, 3H), 1.02 (s, 9H), 0.99 (s, 3H), 0.85 (s, 3H), $0.81(\mathrm{~d}, J=6.6 \mathrm{~Hz}, 3 \mathrm{H}), 0.71(\mathrm{~d}, J=6.6 \mathrm{~Hz}, 3 \mathrm{H}) ;{ }^{13} \mathrm{C} \mathrm{NMR}$ $\left(125 \mathrm{MHz}, \mathrm{CDCl}_{3}\right) \delta 170.8,165.9,157.3\left(\mathrm{q},{ }^{2} J_{\mathrm{C}-\mathrm{F}}=41.6 \mathrm{~Hz}\right.$, $\mathrm{C}=\mathrm{O}), 154.5,135.7,135.5,135.0,134.02,134.0,133.2$, $130.5,129.8,129.5,128.7,127.9,127.8,123.4,114.8(\mathrm{q}$, $\left.{ }^{1} J_{\mathrm{C}-\mathrm{F}}=285.0 \mathrm{~Hz}, \mathrm{CF}_{3}\right), 81.6,78.9,73.6,68.8,54.3,53.3$, 50.7, 44.8, 36.6, 35.9, 35.7, 35.6, 34.0, 33.3, 31.4, 30.9, 29.9, 29.1, 28.5, 27.6, 27.4, 27.2, 26.9, 26.7, 21.6, 19.4, 19.2, 18.4, $16.5,12.2,12.1 ;{ }^{19} \mathrm{~F}$ NMR $\left(470 \mathrm{MHz}, \mathrm{CDC}_{13}\right) \delta-75.0$;
HRMS (ESI) for $\mathrm{C}_{54} \mathrm{H}_{69} \mathrm{~F}_{3} \mathrm{O}_{7} \mathrm{SiNa}[\mathrm{M}+\mathrm{Na}]^{+}$calcd. 937.4662, found 937.4657.

Trifluoroacetate 13: Primary alcohol 12 (101 mg, 0.17 mmol) was used and the reaction was carried out with 4.50 equiv. of TFAT $(0.78 \mathrm{mmol}, 0.12 \mathrm{~mL})$ and 5 equiv. of 2,6 -ditert-butyl-4-methylpyridine $(0.87 \mathrm{mmol}, 178 \mathrm{mg})$ to provide compound 13 (55 mg, $0.07 \mathrm{mmol}$, 42\%, white floppy solid): ${ }^{1} \mathrm{H}$ NMR $\left(500 \mathrm{MHz}, \mathrm{CDCl}_{3}\right) \delta 8.03(\mathrm{~d}, J=8.0 \mathrm{~Hz}, 2 \mathrm{H}), 7.56$ (t, $J=7.3 \mathrm{~Hz}, 1 \mathrm{H}), 7.44$ (t, $J=7.7 \mathrm{~Hz}, 2 \mathrm{H}), 5.38(\mathrm{~s}, 1 \mathrm{H})$, $5.14(\mathrm{dd}, J=10.6,5.2 \mathrm{~Hz}, 1 \mathrm{H}), 5.07-5.10(\mathrm{~m}, 1 \mathrm{H}), 4.65(\mathrm{~m}$, $1 \mathrm{H}), 3.99$ (dd, $J=10.7,6.6 \mathrm{~Hz}, 1 \mathrm{H}), 3.94$ (dd, $J=10.8,6.6$ $\mathrm{Hz}, 1 \mathrm{H}), 2.61$ (dt, $J=15.9,7.1 \mathrm{~Hz}, 1 \mathrm{H}), 2.07-2.12(\mathrm{~m}, 1 \mathrm{H})$, 2.00-2.05 (m, 1H), 1.97 (s, 3H), 0.97 (s, 3H), 0.83 (s, 3H), $0.81(\mathrm{~d}, J=7.0 \mathrm{~Hz}, 3 \mathrm{H}), 0.75(\mathrm{~d}, J=6.6 \mathrm{~Hz}, 3 \mathrm{H}) ;{ }^{13} \mathrm{C} \mathrm{NMR}$ $\left(125 \mathrm{MHz}, \mathrm{CDCl}_{3}\right) \delta 170.8,165.8,157.6\left(\mathrm{q},{ }^{2} J_{\mathrm{C}-\mathrm{F}}=39.4 \mathrm{~Hz}\right.$, $\mathrm{C}=\mathrm{O}), 157.2\left(\mathrm{q},{ }^{2} J_{\mathrm{C}-\mathrm{F}}=42.2 \mathrm{~Hz}, \mathrm{C}=\mathrm{O}\right), 154.4,133.3,130.6$, $129.6,128.7,123.63,123.61,114.7\left(\mathrm{q},{ }^{1} J_{\mathrm{C}-\mathrm{F}}=285.0 \mathrm{~Hz}\right.$, $\left.\mathrm{CF}_{3}\right), 114.6\left(\mathrm{q},{ }^{1} J_{\mathrm{C}-\mathrm{F}}=284.5 \mathrm{~Hz}, \mathrm{CF}_{3}\right), 80.9,78.7,73.6,72.2$, 54.4, 53.3, 50.6, 44.8, 36.6, 35.9, 35.4, 34.0, 33.3, 32.1, 31.4, $30.9,29.9,28.5,28.4,27.6,27.4,26.8,21.6,18.4,16.1,12.2$, $12.1 ;{ }^{19} \mathrm{~F} \mathrm{NMR}\left(470 \mathrm{MHz}, \mathrm{CDCl}_{3}\right) \delta-75.18,-75.20$; HRMS (ESI) for $\mathrm{C}_{40} \mathrm{H}_{52} \mathrm{~F}_{6} \mathrm{O}_{9}\left[\mathrm{M}+\mathrm{H}_{2} \mathrm{O}\right]^{+}$calcd. 790.3516, found 790.3761 .

Trifluroacetates 15, 16, and 17: TBDPS ether 14 (94 mg, $0.13 \mathrm{mmol}$ ) was used and the reaction was carried out for $1 \mathrm{~h}$ with 3 equiv. of TFAT $(0.40 \mathrm{mmol}, 0.06 \mathrm{~mL})$ and 1.20 equiv. of 2,6-di-tert-butyl-4-methylpyridine ( $0.16 \mathrm{mmol}, 33 \mathrm{mg})$. The reaction was purified by HPLC (Silica column, $250 \times$ $10 \mathrm{~mm} \mathrm{ID,} \mathrm{98 \%} n$-Hexane $/ 2 \%$ EtOAc, $2.0 \mathrm{~mL} / \mathrm{min}, \mathrm{RI}$ detector) to provide compound $15(29 \mathrm{mg}, 0.04 \mathrm{mmol}, 27 \%$, white floppy solid), compound 16 (30 mg, $0.04 \mathrm{mmol}, 28 \%$, white floppy solid), and compound 17 (5.4 mg, $0.01 \mathrm{mmol}$, $5 \%$, white floppy solid):

Trifluoroacetate 15: ${ }^{1} \mathrm{H}$ NMR $\left(500 \mathrm{MHz}, \mathrm{CDCl}_{3}\right) \delta 7.64$ $(\mathrm{d}, J=6.6 \mathrm{~Hz}, 4 \mathrm{H}), 7.35-7.42(\mathrm{~m}, 6 \mathrm{H}), 5.42(\mathrm{~s}, 1 \mathrm{H}), 5.18$ (dd, $J=11.5,5.6 \mathrm{~Hz}, 1 \mathrm{H}), 4.67-4.70(\mathrm{~m}, 1 \mathrm{H}), 3.45(\mathrm{t}, J=3.9$ $\mathrm{Hz}, 1 \mathrm{H}), 3.45$ (t, $J=8.6 \mathrm{~Hz}, 1 \mathrm{H}), 2.44$ (quintet, $J=6.6 \mathrm{~Hz}$, 1H), $2.01(\mathrm{~s}, 3 \mathrm{H}), 1.04(\mathrm{~s}, 9 \mathrm{H}), 1.01(\mathrm{~d}, J=6.9 \mathrm{~Hz}, 3 \mathrm{H}), 0.88$ $(\mathrm{d}, J=6.4 \mathrm{~Hz}, 3 \mathrm{H}), 0.85(\mathrm{~s}, 3 \mathrm{H}), 0.73(\mathrm{~s}, 3 \mathrm{H}) ;{ }^{13} \mathrm{C}$ NMR $(125$ $\left.\mathrm{MHz} \mathrm{CDCl}_{3}\right) \delta 170.8,157.5\left(\mathrm{q},{ }^{2} J_{\mathrm{C}-\mathrm{F}}=42.0 \mathrm{~Hz}, \mathrm{C}=\mathrm{O}\right)$, $155.8,135.8,135.7,134.04,134.01,129.7,127.8,123.6$, $114.9\left(\mathrm{q},{ }^{1} J_{\mathrm{C}-\mathrm{F}}=285.0 \mathrm{~Hz}, \mathrm{CF}_{3}\right), 82.0,73.8,68.8,57.4,55.0$, 47.3, 45.1, 36.8, 36.0, 35.9, 35.7, 35.6, 34.8, 34.3, 34.2, 32.0, $31.3,29.9,28.7,28.6,28.3,27.6,27.0,21.6,21.2,19.4,17.5$, 16.6, 16.1, 12.4; ${ }^{19} \mathrm{~F}$ NMR $\left(470 \mathrm{MHz}, \mathrm{CDCl}_{3}\right) \delta-75.0$; HRMS (ESI) for $\mathrm{C}_{47} \mathrm{H}_{66} \mathrm{~F}_{3} \mathrm{O}_{5} \mathrm{Si}[\mathrm{M}+\mathrm{H}]^{+}$calcd. 795.4632, found 795.4641 .

Trifluoroacetate 16: ${ }^{1} \mathrm{H}$ NMR (500 MHz, $\left.\mathrm{CDCl}_{3}\right) \mathrm{d} 7.65$ $(\mathrm{d}, J=7.1 \mathrm{~Hz}, 4 \mathrm{H}), 7.35-7.42(\mathrm{~m}, 6 \mathrm{H}), 5.26(\mathrm{~d}, J=11.0 \mathrm{~Hz}$, $1 \mathrm{H}), 4.68-4.71(\mathrm{~m}, 1 \mathrm{H}), 3.47(\mathrm{t}, J=2.4 \mathrm{~Hz}, 1 \mathrm{H}), 3.46(\mathrm{t}, J=$ $2.7 \mathrm{~Hz}, 1 \mathrm{H}), 2.21-2.23(\mathrm{~m}, 1 \mathrm{H}), 2.09-2.11(\mathrm{~m}, 1 \mathrm{H}), 2.02(\mathrm{~s}$, $3 \mathrm{H}), 1.06(\mathrm{~s}, 3 \mathrm{H}), 1.05(\mathrm{~s}, 9 \mathrm{H}), 0.92(\mathrm{~d}, J=6.6 \mathrm{~Hz}, 3 \mathrm{H}), 0.82$ $(\mathrm{d}, J=7.1 \mathrm{~Hz}, 3 \mathrm{H}), 0.79(\mathrm{~s}, 3 \mathrm{H}) ;{ }^{13} \mathrm{C} \mathrm{NMR}(125 \mathrm{MHz}$, $\left.\mathrm{CDCl}_{3}\right) \delta 170.8,157.3\left(\mathrm{q},{ }^{2} J_{\mathrm{C}-\mathrm{F}}=41.5 \mathrm{~Hz}, \mathrm{C}=\mathrm{O}\right), 139.2$, $138.3,135.8,135.76,134.0,133.9,129.7,127.8,127.7$, $114.9\left(\mathrm{q},{ }^{1} J_{\mathrm{C}-\mathrm{F}}=285.0 \mathrm{~Hz}, \mathrm{CF}_{3}\right), 82.2,77.0,73.9,68.7,51.8$, 51.81, 44.7, 42.2, 37.1, 36.6, 35.6, 35.63, 34.0, 32.9, 31.6, 
$31.3,29.9,29.5,29.1,27.5,27.0,26.9,25.3,23.2,23.1,21.6$, $19.4,16.8,11.9,9.7 ;{ }^{19} \mathrm{~F}$ NMR $\left(470 \mathrm{MHz}, \mathrm{CDCl}_{3}\right) \delta-75.2$; HRMS (ESI) for $\mathrm{C}_{47} \mathrm{H}_{66} \mathrm{~F}_{3} \mathrm{O}_{5} \mathrm{Si}[\mathrm{M}+\mathrm{H}]^{+}$calcd. 795.4632, found 795.4640 .

Trifluoroacetate 17: ${ }^{1} \mathrm{H}$ NMR $\left(500 \mathrm{MHz}, \mathrm{CDCl}_{3}\right) \delta 7.63$ (d, $J=6.3 \mathrm{~Hz}, 4 \mathrm{H}), 7.34-7.40(\mathrm{~m}, 6 \mathrm{H}), 5.24-5.26(\mathrm{~m}, 2 \mathrm{H})$, $4.62-4.70(\mathrm{~m}, 1 \mathrm{H}), 3.43(\mathrm{~d}, J=5.9 \mathrm{~Hz}, 1 \mathrm{H}), 2.01(\mathrm{~s}, 3 \mathrm{H})$, $1.04(\mathrm{~s}, 3 \mathrm{H}), 1.02(\mathrm{~s}, 9 \mathrm{H}), 0.88(\mathrm{~d}, J=6.9 \mathrm{~Hz}, 3 \mathrm{H}), 0.85(\mathrm{~d}, J$ $=7.1 \mathrm{~Hz}, 3 \mathrm{H}), 0.75(\mathrm{~s}, 3 \mathrm{H}) ;{ }^{13} \mathrm{C} \mathrm{NMR}\left(125 \mathrm{MHz}, \mathrm{CDCl}_{3}\right) \delta$ $170.9,157.3\left(\mathrm{q},{ }^{2} J_{\mathrm{C}-\mathrm{F}}=41.0 \mathrm{~Hz}, \mathrm{C}=\mathrm{O}\right), 150.8,135.81$, $135.79,134.1,134.0,129.8,127.81,127.80,116.3,114.9(\mathrm{q}$, $\left.{ }^{1} J_{\mathrm{C}-\mathrm{F}}=285.0 \mathrm{~Hz}, \mathrm{CF}_{3}\right), 82.1,73.8,68.8,50.1,48.4,46.6$, $45.0,44.5,39.0,37.0,35.65,35.61,35.3,34.3,32.7,29.9$, $29.5,29.4,28.1,27.4,27.0,26.97,25.6,21.7,19.5,16.8$, 12.3, 9.9; ${ }^{19} \mathrm{~F}$ NMR (470 MHz, $\left.\mathrm{CDCl}_{3}\right) \delta-75.2$; HRMS (ESI) for $\mathrm{C}_{47} \mathrm{H}_{66} \mathrm{~F}_{3} \mathrm{O}_{5} \mathrm{Si}[\mathrm{M}+\mathrm{H}]^{+}$calcd. 795.4632, found 795.4644.

Secondary Alcohol 18: Trifluoroacetate 11 (305 mg, 0.33 mmol) was stirred with $\mathrm{K}_{2} \mathrm{CO}_{3}(92 \mathrm{mg}, 0.67 \mathrm{mmol}, 2$ equiv.) and thiourea ( $63 \mathrm{mg}, 0.83 \mathrm{mmol}, 2.50$ equiv.) in $6.70 \mathrm{~mL}$ of $\mathrm{EtOH}$ at room temperature for $4.5 \mathrm{~h}$. The reaction was quenched with EtOAc/ $\mathrm{H}_{2} \mathrm{O}$, extracted with EtOAc three times. The extract was washed with brine, dried over $\mathrm{Na}_{2} \mathrm{SO}_{4}$, filtered, and concentrated in vacuo. The crude residue was purified by flash column chromatography (EtOAc/ $n$-Hexane $=1: 6$ to $1: 3)$ to give secondary alcohol $\mathbf{1 8}(271 \mathrm{mg}, 0.33$ mmol, 99\%) as white solid; ${ }^{1} \mathrm{H}$ NMR $\left(500 \mathrm{MHz}, \mathrm{CDCl}_{3}\right) \delta$ 8.02 (d, $J=7.6 \mathrm{~Hz}, 2 \mathrm{H}), 7.64$ (d, $J=6.6 \mathrm{~Hz}, 4 \mathrm{H}), 7.34-7.43$ (m, 9H), 5.49 (s, 1H), 5.15 (dd, $J=5.7,4.5 \mathrm{~Hz}, 1 \mathrm{H}), 4.66-$ $4.71(\mathrm{~m}, 1 \mathrm{H}), 3.54(\mathrm{t}, J=7.6 \mathrm{~Hz}, 1 \mathrm{H}), 3.44(\mathrm{dt}, J=5.3,4.4$ $\mathrm{Hz}, 1 \mathrm{H}$ ), 3.29 (t, $J=8.0 \mathrm{~Hz}, 1 \mathrm{H}$ ), 2.3 (quintet, $J=7.4 \mathrm{~Hz}$, $1 \mathrm{H}), 2.15-2.18(\mathrm{~m}, 1 \mathrm{H}), 2.01-2.09(\mathrm{~m}, 1 \mathrm{H}), 2.00(\mathrm{~s}, 3 \mathrm{H})$, $1.04(\mathrm{~s}, 3 \mathrm{H}), 1.02(\mathrm{~s}, 9 \mathrm{H}), 0.85(\mathrm{~s}, 3 \mathrm{H}), 0.80(\mathrm{~d}, J=6.4 \mathrm{~Hz}$, $3 \mathrm{H}), 0.75(\mathrm{~d}, J=6.4 \mathrm{~Hz}, 3 \mathrm{H}) ;{ }^{13} \mathrm{C} \mathrm{NMR}\left(125 \mathrm{MHz}, \mathrm{CDCl}_{3}\right)$ $\delta 170.8,165.9,157.5,135.8,134.24,134.22,133.2,130.6$, $129.65,129.60,128.6,127.7,123.0,78.6,73.7,73.6,69.2$, 54.6, 53.3, 50.7, 44.8, 39.8, 36.7, 36.1, 35.9, 34.0, 33.4, 31.4, $30.9,30.6,28.5,28.4,27.6,27.5,27.0,21.6,19.4,18.1,17.0$, 13.0, 12.3; HRMS (ESI) for $\mathrm{C}_{52} \mathrm{H}_{70} \mathrm{O}_{6} \mathrm{SiNa}[\mathrm{M}+\mathrm{Na}]^{+}$calcd. 841.4839 , found 841.4815.

TBS Ether 19: To a solution of secondary alcohol 18 (268 $\mathrm{mg}, 0.33 \mathrm{mmol})$, triethylamine $(0.09 \mathrm{~mL}, 0.65 \mathrm{mmol}, 2$ equiv.) in $\mathrm{CH}_{2} \mathrm{Cl}_{2}(6.5 \mathrm{~mL})$ was added TBSOTf $(0.11 \mathrm{~mL}$, $0.49 \mathrm{mmol}, 1.50$ equiv.) at room temperature. The reaction was stirred for $0.5 \mathrm{~h}$. The reaction was diluted with $\mathrm{CH}_{2} \mathrm{Cl}_{2}$, worked up with sat. $\mathrm{NaHCO}_{3}$. The organic layer was washed with brine, dried over $\mathrm{Na}_{2} \mathrm{SO}_{4}$, filtered, and concentrated in vacuo. The crude residue was purified by flash chromatography $(\mathrm{EtOAc} / n$-Hexane $=1: 15$ to $1: 10)$ to give TBS ether $19(287 \mathrm{mg}, 0.31 \mathrm{mmol}, 94 \%)$ as white floppy solid; ${ }^{1} \mathrm{H}$ $\operatorname{NMR}\left(500 \mathrm{MHz}, \mathrm{CDCl}_{3}\right) \delta 7.99(\mathrm{~d}, J=7.5 \mathrm{~Hz}, 2 \mathrm{H}), 7.68(\mathrm{~d}$, $J=5.9 \mathrm{~Hz}, 4 \mathrm{H}), 7.40-7.45(\mathrm{~m}, 6 \mathrm{H}), 7.26-7.34(\mathrm{~m}, 4 \mathrm{H}), 5.54$ (s, $1 \mathrm{H}), 5.15$ (dd, $J=10.2,4.9 \mathrm{~Hz}, 1 \mathrm{H}), 4.69-4.73(\mathrm{~m}, 1 \mathrm{H})$, 3.59 (d, $J=4.4 \mathrm{~Hz}, 1 \mathrm{H}), 3.43(\mathrm{dd}, J=9.5,5.7 \mathrm{~Hz}, 1 \mathrm{H}), 3.23$ (t, $J=7.8 \mathrm{~Hz}, 1 \mathrm{H}$ ), 2.35 (quintet, $J=6.4 \mathrm{~Hz}, 1 \mathrm{H}$ ), 2.02 (s, $3 \mathrm{H}), 1.06(\mathrm{~s}, 9 \mathrm{H}), 0.98(\mathrm{~s}, 3 \mathrm{H}), 0.87(\mathrm{~s}, 3 \mathrm{H}), 0.84(\mathrm{~s}, 9 \mathrm{H})$, $0.80(\mathrm{~d}, J=5.6 \mathrm{~Hz}, 3 \mathrm{H}), 0.79(\mathrm{~d}, J=6.1 \mathrm{~Hz}, 3 \mathrm{H}),-0.02(\mathrm{~s}$,
$3 \mathrm{H}),-0.06(\mathrm{~s}, 3 \mathrm{H}) ;{ }^{13} \mathrm{C} \mathrm{NMR}\left(125 \mathrm{MHz}, \mathrm{CDCl}_{3}\right) \delta 170.8$, $166.0,156.1,135.8,134.3,133.0,130.8,129.7,129.6$, $128.5,127.8,123.1,79.1,76.1,73.7,69.5,54.3,53.4,50.8$, 44.9, 36.8, 36.7, 36.5, 35.9, 34.1, 33.4, 32.4, 31.5, 31.0, 28.6, 27.7, 27.6, 27.5, 27.1, 27.05, 26.2, 26.1, 21.6, 19.5, 19.1, $18.2,17.0,12.54,12.48,12.3,12.2$; HRMS (ESI) for $\mathrm{C}_{58} \mathrm{H}_{84} \mathrm{O}_{6} \mathrm{Si}_{2}\left[\mathrm{M}+\mathrm{H}_{2} \mathrm{O}\right]^{+}$calcd. 950.5912 , found 950.6161 .

Diol 20: To a solution of TBS ether 19 (128 mg, 0.14 mmol) in pyridine/THF $(1.4 \mathrm{~mL} / 2.8 \mathrm{~mL})$ was added $\mathrm{OsO}_{4}$ ( $2.5 \mathrm{wt} \%$ in tBuOH, $3.10 \mathrm{~mL}, 0.30 \mathrm{mmol}, 2.20$ equiv.) at $78{ }^{\circ} \mathrm{C}$. The reaction was stirred for $26 \mathrm{~h}$ with temperature allowed to reach room temperature. After dilution with EtOAc, the reaction was treated with $1 \mathrm{~N}$ aq. $\mathrm{HCl}$ solution to remove pyridine. The resulting solution was neutralized with sat. $\mathrm{NaHCO}_{3}$, dried over $\mathrm{Na}_{2} \mathrm{SO}_{4}$, filtered, and concentrated in vacuo. To the concentrate was added sat. $\mathrm{NaHSO}_{3} / \mathrm{THF}$ (1:1) and the solution was heated for $3.5 \mathrm{~h}$ at $60{ }^{\circ} \mathrm{C}$. The reaction was diluted with EtOAc, worked up with water. The organic layer was washed with brine, dried over $\mathrm{Na}_{2} \mathrm{SO}_{4}$, filtered, and concentrated in vacuo. The crude residue was purified by flash chromatography $(\mathrm{EtOAc} / n$-Hexane $=1: 6)$ to give diol 20 (129 $\mathrm{mg}, 0.13 \mathrm{mmol}, 98 \%)$ as white floppy solid; ${ }^{1} \mathrm{H}$ NMR $\left(500 \mathrm{MHz}, \mathrm{CDCl}_{3}\right) \delta 8.00(\mathrm{~d}, J=7.4 \mathrm{~Hz}$, 2H), $7.64(\mathrm{~d}, J=6.6 \mathrm{~Hz}, 4 \mathrm{H}), 7.53(\mathrm{t}, J=7.4 \mathrm{~Hz}, 1 \mathrm{H}), 7.34-$ $7.42(\mathrm{~m}, 8 \mathrm{H}), 5.31(\mathrm{dd}, J=10.9,4.8 \mathrm{~Hz}, 1 \mathrm{H}), 4.65-4.69(\mathrm{~m}$, $1 \mathrm{H}), 4.30(\mathrm{t}, J=6.1 \mathrm{~Hz}, 1 \mathrm{H}), 3.74(\mathrm{~s}, 1 \mathrm{H}), 7.03(\mathrm{~d}, J=5.2$ $\mathrm{Hz}, 1 \mathrm{H}), 3.47$ (dd, $J=9.8,5.6 \mathrm{~Hz}, 1 \mathrm{H}), 3.42$ (dd, $J=9.6,6.2$ $\mathrm{Hz}, 1 \mathrm{H}), 3.09(\mathrm{~s}, 1 \mathrm{H}), 1.99(\mathrm{~s}, 3 \mathrm{H}), 1.04(\mathrm{~s}, 9 \mathrm{H}), 0.95(\mathrm{~s}, 3 \mathrm{H})$, $0.88(\mathrm{~d}, J=6.6 \mathrm{~Hz}, 3 \mathrm{H}), 0.82(\mathrm{~s}, 9 \mathrm{H}), 0.817(\mathrm{~s}, 3 \mathrm{H}), 0.74(\mathrm{~d}$, $J=6.9 \mathrm{~Hz}, 3 \mathrm{H}), 0.00(\mathrm{~s}, 3 \mathrm{H}),-0.003(\mathrm{~s}, 3 \mathrm{H}) ;{ }^{13} \mathrm{C}$ NMR $(125$ $\left.\mathrm{MHz}, \mathrm{CDCl}_{3}\right) \delta 170.8,166.7,135.82,135.81,134.24,134.22$, 133.2, 131.0, 129.8, 129.7, 128.6, 127.8, 84.4, 78.0, 74.9, 74.7, 73.6, 69.2, 53.4, 52.0, 47.7, 44.6, 42.5, 36.7, 36.1, 35.6, $34.5,34.0,32.9,31.5,30.9,29.9,28.6,27.8,27.4,27.1,26.1$, 21.6, 19.5, 18.2, 17.0, 12.4, 12.2, 11.2, -4.1; HRMS (EI) for $\mathrm{C}_{58} \mathrm{H}_{87} \mathrm{O}_{8} \mathrm{Si}_{2}[\mathrm{M}+\mathrm{H}]^{+}$calcd. 967.5939, found 967.5940 .

Ketone 22: Solid TPAP, tetrapropylammonium perruthenate $(12 \mathrm{mg}, 0.03 \mathrm{mmol}, 0.25$ equiv.) was added in one portion to a stirred solution of diol $20(129 \mathrm{mg}, 0.13 \mathrm{mmol})$, NMO, 4-methylmorpholine $N$-oxide ( $47 \mathrm{mg}, 0.40 \mathrm{mmol}, 3$ equiv.) and powdered molecular sieves ( $13 \mathrm{mg}, 4 \AA$, activated) in $\mathrm{CH}_{2} \mathrm{Cl}_{2}(2.7 \mathrm{~mL})$ at room temperature. On completion, the reaction was filtered through a short celite pad $(5 \mathrm{~cm})$, and the filtrate was concentrated in vacuo. The crude residue was purified by flash chromatography $(\mathrm{EtOAc} / n-\mathrm{Hexane}=$ $1: 6$ to $1: 4$ ) to provide ketone 22 ( $94 \mathrm{mg}, 0.10 \mathrm{mmol}, 71 \%$ overall from TBS ether 19) as white solid; ${ }^{1} \mathrm{H}$ NMR (500 $\left.\mathrm{MHz}, \mathrm{CDCl}_{3}\right) \delta 7.99(\mathrm{~d}, J=7.8 \mathrm{~Hz}, 2 \mathrm{H}), 7.64(\mathrm{~d}, J=6.4 \mathrm{~Hz}$, 4H), $7.51(\mathrm{t}, J=7.0 \mathrm{~Hz}, 1 \mathrm{H}), 7.34-7.40(\mathrm{~m}, 8 \mathrm{H}), 5.41$ (dd, $J$ $=10.7,4.6 \mathrm{~Hz}, 1 \mathrm{H}), 4.66-4.70(\mathrm{~m}, 1 \mathrm{H}), 4.12(\mathrm{~s}, 1 \mathrm{H}), 3.42-$ $3.46(\mathrm{~m}, 2 \mathrm{H}), 3.35(\mathrm{dd}, J=9.5,6.9 \mathrm{~Hz}, 1 \mathrm{H}), 2.35(\mathrm{dd}, J=$ $17.2,6.6 \mathrm{~Hz}, 1 \mathrm{H}), 2.29(\mathrm{t}, J=6.6 \mathrm{~Hz}, 1 \mathrm{H}), 1.99(\mathrm{~s}, 3 \mathrm{H}), 1.24$ (s, 3H), $1.12(\mathrm{~s}, 3 \mathrm{H}), 1.02(\mathrm{~s}, 9 \mathrm{H}), 0.84(\mathrm{~s}, 3 \mathrm{H}), 0.82(\mathrm{~d}, J=$ $10.5 \mathrm{~Hz}, 3 \mathrm{H}), 0.81(\mathrm{~s}, 9 \mathrm{H}), 0.77$ (d, $J=6.8 \mathrm{~Hz}, 3 \mathrm{H}),-0.03(\mathrm{~s}$, $3 \mathrm{H}),-0.06$ (s, 3H); ${ }^{13} \mathrm{C}$ NMR $\left(125 \mathrm{MHz}, \mathrm{CDCl}_{3}\right) \delta 214.1$, $170.8,165.8,135.8,134.2,133.2,130.8,129.7,129.6$, 128.6, 127.7, 84.3, 74.0, 73.53, 73.52, 69.5, 51.7, 51.2, 44.5, 
$44.0,40.5,37.2,36.5,36.3,35.7,33.9,33.7,32.2,31.6,29.9$, 28.5, 28.4, 27.4, 27.1, 27.0, 26.1, 21.6, 19.5, 18.2, 16.7, 12.2, 12.0, 11.2, -3.8, -4.3; HRMS (EI) for $\mathrm{C}_{58} \mathrm{H}_{85} \mathrm{O}_{8} \mathrm{Si}_{2}[\mathrm{M}+\mathrm{H}]^{+}$ calcd. 965.5783 , found 965.5803 .

Aldehyde 21: To a solution of diol 20 (12 mg, $0.01 \mathrm{mmol})$ in $\mathrm{CH}_{2} \mathrm{Cl}_{2}(0.25 \mathrm{~mL})$ was added Dess-Martin periodinane (11 mg, $0.03 \mathrm{mmol}, 2$ equiv.) and stirred for $2 \mathrm{~h}$ at room temperature. The reaction was diluted with $\mathrm{CH}_{2} \mathrm{Cl}_{2}$, worked up with sat. $\mathrm{NaHCO}_{3}$. The organic layer was washed with brine, dried over $\mathrm{Na}_{2} \mathrm{SO}_{4}$, filtered, and concentrated in vacuo. The crude residue was purified by flash chromatography $($ EtOAc/ $n$-Hexane $=1: 6)$ to give aldehyde 21 (12 mg, 0.01 mmol, 95\%) as white floppy solid; ${ }^{1} \mathrm{H}$ NMR (500 MHz, $\left.\mathrm{CDCl}_{3}\right) \delta 9.69(\mathrm{~d}, J=1.7 \mathrm{~Hz}, 1 \mathrm{H}), 7.86(\mathrm{~d}, J=8.0 \mathrm{~Hz}, 2 \mathrm{H})$, $7.60-7.62(\mathrm{~m}, 4 \mathrm{H}), 7.35-7.42(\mathrm{~m}, 7 \mathrm{H}), 7.30(\mathrm{t}, J=7.5 \mathrm{~Hz}$, $1 \mathrm{H}), 7.18-7.24(\mathrm{~m}, 2 \mathrm{H}), 5.21(\mathrm{dd}, J=11.2,4.2 \mathrm{~Hz}, 1 \mathrm{H})$, 4.66-4.71 (m, 1H), 3.60-3.62 (m, 1H), $3.51(\mathrm{t}, J=6.3 \mathrm{~Hz}$, $1 \mathrm{H}), 3.20(\mathrm{dd}, J=9.4,5.3 \mathrm{~Hz}, 1 \mathrm{H}), 3.04(\mathrm{dd}, J=9.4,7.0 \mathrm{~Hz}$, $1 \mathrm{H}), 2.45$ (t, $J=9.4 \mathrm{~Hz}, 1 \mathrm{H}), 2.20-2.30(\mathrm{~m}, 2 \mathrm{H}), 1.99$ (s, $3 \mathrm{H}), 1.32(\mathrm{~s}, 3 \mathrm{H}), 1.00(\mathrm{~s}, 9 \mathrm{H}), 0.84(\mathrm{~s}, 9 \mathrm{H}), 0.79(\mathrm{~s}, 3 \mathrm{H})$, $0.78(\mathrm{~d}, J=8.5 \mathrm{~Hz}, 3 \mathrm{H}), 0.70(\mathrm{~d}, J=6.6 \mathrm{~Hz}, 3 \mathrm{H}), 0.12(\mathrm{~s}$, $3 \mathrm{H}), 0.01(\mathrm{~s}, 3 \mathrm{H}) ;{ }^{13} \mathrm{C} \mathrm{NMR}\left(125 \mathrm{MHz}, \mathrm{CDCl}_{3}\right) \delta 216.2$, $200.9,170.8,165.4,135.8,134.2,133.4,130.0,129.7$, $129.65,129.5,128.6,127.76,127.74,77.4,77.0,73.4,72.5$, 69.5, 57.4, 50.4, 46.8, 45.9, 44.0, 40.4, 37.4, 36.7, 36.1, 35.7, $33.8,31.7,29.1,28.7,28.4,27.3,27.0,26.1,26.0,21.6,19.5$, $18.3,16.4,12.3,12.2,10.8,-3.7,-4.4$; HRMS (ESI) for $\mathrm{C}_{58} \mathrm{H}_{85} \mathrm{O}_{8} \mathrm{Si}_{2}[\mathrm{M}+\mathrm{H}]^{+}$calcd. 965.5783, found 965.5792 .

Hemiketal 23: TBAF, tetrabutylammonium fluoride, 1.0 $\mathrm{M}$ solution in THF ( $0.29 \mathrm{~mL}, 0.29 \mathrm{mmol}, 7.50$ equiv.) was added to a stirred solution of ketone $22(37 \mathrm{mg}, 0.04 \mathrm{mmol})$ in THF $(3.8 \mathrm{~mL})$. The reaction was stirred at room temperature for $24 \mathrm{~h}$. On completion the reaction mixture was worked up with sat. $\mathrm{NaHCO}_{3} / \mathrm{EtOAc}$, washed with brine, dried over $\mathrm{Na}_{2} \mathrm{SO}_{4}$. After removal of solvent under reduced pressure, the reaction mixture was purified by flash column chromatography $(\mathrm{EtOAc} / n$-Hexane $=1: 1)$ to provide hemiketal 21 (23 mg, $0.04 \mathrm{mmol}, 100 \%)$ as white solid; ${ }^{1} \mathrm{H}$ NMR (500 MHz, $\left.\mathrm{CDCl}_{3}\right) \delta 8.01(\mathrm{~d}, J=7.8 \mathrm{~Hz}, 2 \mathrm{H}), 7.58(\mathrm{t}$, $J=7.2 \mathrm{~Hz}, 1 \mathrm{H}), 7.44(\mathrm{t}, J=7.0 \mathrm{~Hz}, 2 \mathrm{H}), 5.25(\mathrm{dd}, J=11.2$, $2.9 \mathrm{~Hz}, 1 \mathrm{H}), 4.64-4.68(\mathrm{~m}, 1 \mathrm{H}), 4.47(\mathrm{~s}, 1 \mathrm{H}), 4.19(\mathrm{~s}, 1 \mathrm{H})$, $3.68(\mathrm{t}, J=7.8 \mathrm{~Hz}, 1 \mathrm{H}), 3.43(\mathrm{dd}, J=16.4,9.8 \mathrm{~Hz}, 1 \mathrm{H}), 3.39$ (dd, $J=15.8,11.0 \mathrm{~Hz}, 1 \mathrm{H}), 1.99(\mathrm{~s}, 3 \mathrm{H}), 1.23$ (s, 3H), 1.00 $(\mathrm{s}, 3 \mathrm{H}), 0.86(\mathrm{~s}, 3 \mathrm{H}), 0.85(\mathrm{~d}, J=7.8 \mathrm{~Hz}, 3 \mathrm{H}), 0.58(\mathrm{~d}, J=$ $6.1 \mathrm{~Hz}, 3 \mathrm{H}) ;{ }^{13} \mathrm{C} \mathrm{NMR}\left(125 \mathrm{MHz}, \mathrm{CDCl}_{3}\right) \delta 170.6,167.7$, $133.7,130.0,129.9,128.8,112.0,89.1,87.8,75.8,73.5$, 68.3, 52.3, 50.9, 50.7, 44.6, 40.9, 39.1, 36.7, 35.8, 35.7, 34.4, $34.0,31.4,30.5,29.9,29.86,28.5,27.5,27.2,21.6,16.7$, 12.3, 12.1, 12.0; HRMS (ESI) for $\mathrm{C}_{36} \mathrm{H}_{51} \mathrm{O}_{7}[\mathrm{M}-\mathrm{OH}]^{+}$calcd. 595.3634, found 595.3636.

Primary Alcohol 24: To a solution of hemiketal 23 (18 $\mathrm{mg}, 0.03 \mathrm{mmol})$ and $\mathrm{Et}_{3} \mathrm{SiH}(0.03 \mathrm{~mL}, 0.18 \mathrm{mmol}, 6$ equiv. in $\mathrm{CH}_{2} \mathrm{Cl}_{2}(0.59 \mathrm{~mL})$ was added $\mathrm{BF}_{3} . \mathrm{OEt}_{2}(0.01 \mathrm{~mL}, 0.06$ mmol, 2 equiv.) at $0{ }^{\circ} \mathrm{C}$ and stirred for $1.3 \mathrm{~h}$ at the same temperature. The reaction was diluted with $\mathrm{CH}_{2} \mathrm{Cl}_{2}$, worked up with sat. $\mathrm{NaHCO}_{3}$. The organic layer was washed with brine, dried over $\mathrm{Na}_{2} \mathrm{SO}_{4}$, filtered, and concentrated in vacuo.
The crude residue was purified by flash chromatography $($ EtOAc $/ n$-Hexane $=1: 1)$ to give primary alcohol $24(10 \mathrm{mg}$, $0.02 \mathrm{mmol}, 55 \%)$ as white floppy solid; ${ }^{1} \mathrm{H} \mathrm{NMR}(500 \mathrm{MHz}$, $\left.\mathrm{CDCl}_{3}\right) \delta 8.01(\mathrm{~d}, J=7.8 \mathrm{~Hz}, 2 \mathrm{H}), 7.58(\mathrm{t}, J=7.5 \mathrm{~Hz}, 1 \mathrm{H})$, $7.44(\mathrm{t}, J=7.6 \mathrm{~Hz}, 2 \mathrm{H}), 5.22(\mathrm{dd}, J=11.3,4.4 \mathrm{~Hz}, 1 \mathrm{H})$, 4.64-4.68 (m, 1H), $3.91(\mathrm{t}, J=5.6 \mathrm{~Hz}, 1 \mathrm{H}), 3.37-3.45(\mathrm{~m}$, 4H), 2.13 (quintet, $J=6.7 \mathrm{~Hz}, 1 \mathrm{H}), 1.99(\mathrm{~s}, 3 \mathrm{H}), 0.94(\mathrm{~s}$, $3 \mathrm{H}), 0.88$ (s, 3H), 0.85 (d, $J=6.6 \mathrm{~Hz}, 3 \mathrm{H}), 0.59$ (d, $J=6.6$ $\mathrm{Hz}, 3 \mathrm{H}) ;{ }^{13} \mathrm{C}$ NMR $\left(125 \mathrm{MHz}, \mathrm{CDCl}_{3}\right) \delta 170.8,167.8$, $133.7,130.1,129.9,128.8,90.4,89.9,75.7,73.6,68.3,52.5$, 51.7, 50.6, 44.6, 41.7, 36.8, 35.8, 35.7, 34.8, 34.0, 31.5, 30.9, 30.6, 29.9, 28.5, 27.5, 27.4, 21.6, 16.7, 12.4, 11.8, 11.5; HRMS (ESI) for $\mathrm{C}_{36} \mathrm{H}_{53} \mathrm{O}_{7}[\mathrm{M}+\mathrm{H}]^{+}$calcd. 597.3791, found 597.3788 .

C12- $\beta-\mathrm{OBz}, \mathrm{C17}-\alpha-\mathrm{OH}$ Rockogenin Acetate 25: Primary alcohol $22(10 \mathrm{mg}, 0.02 \mathrm{mmol})$ was stirred with diacetoxy iodobenzene ( $11 \mathrm{mg}, 0.04 \mathrm{mmol}, 2.20$ equiv.) and iodine ( $4 \mathrm{mg}, 0.02 \mathrm{mmol}$, 1 equiv.) in $3.3 \mathrm{~mL}$ of cyclohexane $/ \mathrm{CH}_{2} \mathrm{Cl}_{2}$ (1:1 mixture) for $6 \mathrm{~h}$ at $0{ }^{\circ} \mathrm{C}$. The reaction was diluted with $\mathrm{CH}_{2} \mathrm{Cl}_{2}$, and quenched with sat. $\mathrm{Na}_{2} \mathrm{~S}_{2} \mathrm{O}_{3}$. The organic layer was washed with brine, dried over $\mathrm{Na}_{2} \mathrm{SO}_{4}$, filtered, and concentrated in vacuo. The crude residue was purified by flash column chromatography (EtOAc/n-Hexane $=1: 3)$ to give secondary alcohol $23(9 \mathrm{mg}, 0.01 \mathrm{mmol}, 90 \%)$ as white solid; ${ }^{1} \mathrm{H}$ NMR $\left(500 \mathrm{MHz}, \mathrm{CDCl}_{3}\right) \delta 8.01(\mathrm{~d}, J=$ $7.9 \mathrm{~Hz}, 2 \mathrm{H}), 7.53$ (t, $J=7.4 \mathrm{~Hz}, 1 \mathrm{H}), 7.42$ (t, $J=7.5 \mathrm{~Hz}, 2 \mathrm{H})$, $5.34(\mathrm{dd}, J=11.1,4.5 \mathrm{~Hz}, 1 \mathrm{H}), 4.61-4.66(\mathrm{~m}, 1 \mathrm{H}), 4.01(\mathrm{t}, J$ $=6.8 \mathrm{~Hz}, 1 \mathrm{H}), 3.45(\mathrm{~d}, J=11.0 \mathrm{~Hz}, 1 \mathrm{H}), 3.31(\mathrm{t}, J=11.4 \mathrm{~Hz}$, $1 \mathrm{H}), 2.65(\mathrm{~s}, 1 \mathrm{H}), 2.10-2.15(\mathrm{~m}, 1 \mathrm{H}), 1.99(\mathrm{~s}, 3 \mathrm{H}), 0.98(\mathrm{~s}$, $3 \mathrm{H}), 0.85$ (s, 3H), 0.75 (d, $J=5.8 \mathrm{~Hz}, 3 \mathrm{H}), 0.60$ (d, $J=6.6$ $\mathrm{Hz}, 3 \mathrm{H}) ;{ }^{13} \mathrm{C} \mathrm{NMR}\left(125 \mathrm{MHz}, \mathrm{CDCl}_{3}\right) \delta 170.8,166.5$, $133.2,130.8,129.9,128.6,110.0,89.8,89.4,74.9,73.6$, 67.0, 52.4, 51.6, 49.5, 44.9, 44.6, 36.8, 35.8, 34.6, 34.0, 31.6, $31.4,30.8,30.2,28.5,28.4,27.5,27.2,21.6,17.3,12.3,12.2$, 7.8; HRMS (ESI) for $\mathrm{C}_{36} \mathrm{H}_{51} \mathrm{O}_{7}[\mathrm{M}+\mathrm{H}]^{+}$calcd. 595.3635, found 595.3632.

\section{Results and Discussion}

Herein we describe an efficient method to open tetrahydrofuranyl subunits in steroids using TFAT (trifluoroacetyl trifluoromethanesulfonate) and an efficient synthesis of $\mathrm{C} 12-\beta-\mathrm{OBz}, \mathrm{C} 17-\alpha-\mathrm{OH}$ rockogenin acetate.

Since the $\mathrm{C} 17$ position is placed in a sterically demanding environment, it is envisaged that access to the $\mathrm{C} 17$ position can be realized by E-ring opening of steroids. During preliminary studies under various reaction conditions for tetrahydrofuran ring opening, it was discovered that treatment of 3 with TFAT alone in $\mathrm{CH}_{2} \mathrm{Cl}_{2}$ at $-35^{\circ} \mathrm{C}$ afforded an E-ring opened compound $\mathbf{4}$ in $70 \%$ yield, but the product also indicated that acid-promoted subsequent double bond migration to the thermodynamically more stable tri-substituted position occurred owing to the triflic acid generated during the reaction (Scheme 2). It was, therefore, anticipated that addition of a TFAT-compatible acid scavenger, 2,6-di-tertbutyl-4-methylpyridine would prevent the unwanted double bond migration following E-ring cleavage (Scheme 2). ${ }^{6}$ 


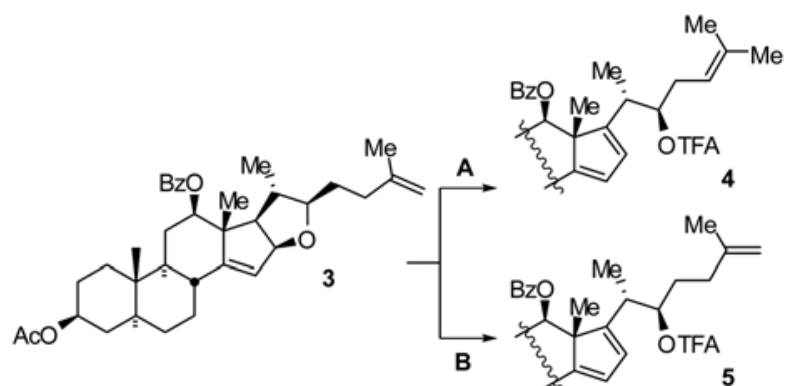

condition $\mathbf{A}=3$ equiv. TFAT only, $150 \mathrm{~min} .,-35^{\circ} \mathrm{C}, 70 \%$ yield.

condition $\mathbf{B}=1.5$ equiv. TFAT, 1.2 equiv. 2,6-di-tert-butyl-4methylpyridine, $60 \mathrm{~min} .,-35^{\circ} \mathrm{C}, 97 \%$ yield.

Scheme 2. Acid-promoted double bond migration.

Hence, the reaction of TFAT in combination with an acid scavenger, 2,6-di-tert-butyl-4-methylpyridine prevents the unwanted double bond migration providing the desired product 5 in $97 \%$ yield (Scheme 2).

This method was also applied to other acid sensitive substrates. Thus, the above reaction condition readily transformed TBDPS ether 6 to the corresponding diene 7 in $91 \%$ yield (entry 1 , Table 1$)^{7}$

Surprisingly, application of the same reaction condition to the non-allylic C14,15-dihydro steroid 8 with TFAT and 2,6di-tert-butyl-4-methylpyridine gave compound 9 in $61 \%$ yield (entry 2, Table 1). Likewise, reaction of TBDPS ether $\mathbf{1 0}$ provided the corresponding E-ring opened compound $\mathbf{1 1}$ in $68 \%$ yield (entry 3 , Table 1$){ }^{8}$

Furthermore, di-trifluoroacetate $\mathbf{1 3}$ was obtained by consecutive transformations of trifluoroacetylation of primary alcohol 12 to mono-trifluoroacetate, followed by E-ring opening (entry 4, Table 1).

In stark contrast, the above reaction condition triggered Wagner-Meerwein rearrangement in the reaction of TBDPS

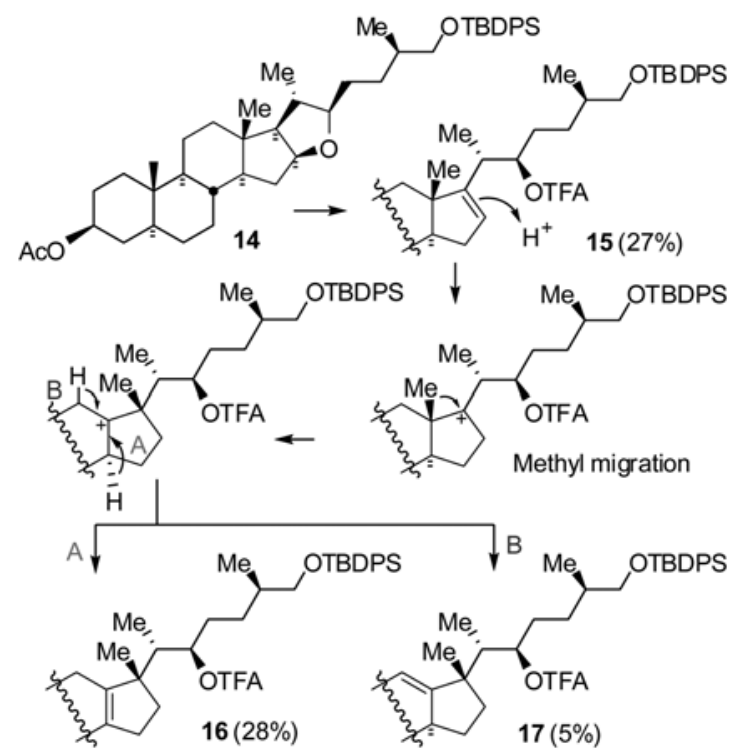

Scheme 3. Wagner-Meerwein rearrangement of C18 methyl group.

ether 14 which doesn't have C12- $\beta$-OBz (Scheme 3). ${ }^{9}$ Thus, the $\mathrm{C} 18$ angular methyl group migrated to the adjacent $\mathrm{C} 17-$ $\beta$ position providing the corresponding olefinic steroids $\mathbf{1 6}$ (28\%), and $17(5 \%)$, accompanied by the E-ring opened product 15 in $27 \%$ yield (Scheme 3). ${ }^{10,11}$

Inspired by the results, synthesis of $\mathrm{C} 12-\beta-\mathrm{OBz}, \mathrm{C} 17-\alpha-$ $\mathrm{OH}$ rockogenin acetate was attempted as a synthetic application of the newly developed TFAT-promoted E-ring opening method to install a hydroxyl group into the $\mathrm{C} 17$ position (Scheme 4).

Mild deprotection of trifluoroacetate 11 with $\mathrm{K}_{2} \mathrm{CO}_{3}$ and thiourea, followed by TBS protection provided compound 19 in $93 \%$ overall yield. ${ }^{12}$ After stoichiometric dihydroxylation with $\mathrm{OsO}_{4}$, oxidation of the resulting diol $\mathbf{2 0}$ to the

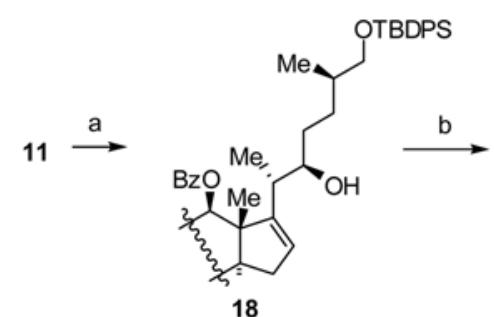

18

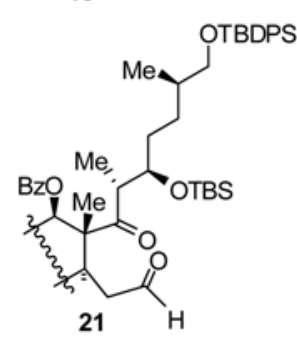

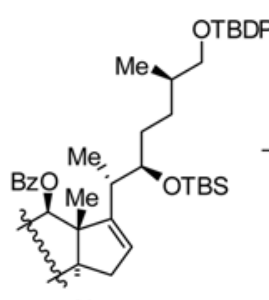

19

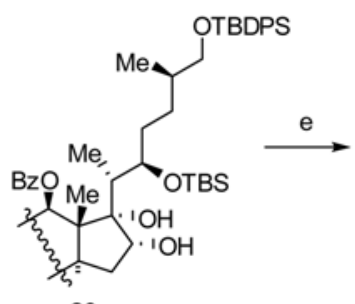

20

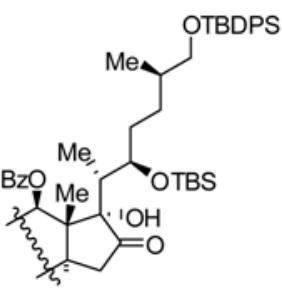

22

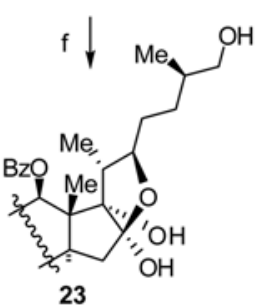

23

25

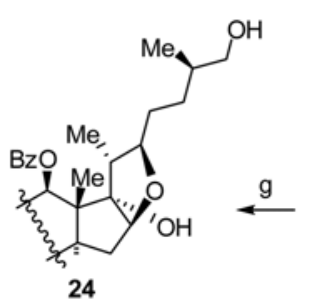

a. 2 equiv. $\mathrm{K}_{2} \mathrm{CO}_{3}, 2.5$ equiv. thiourea, $\mathrm{EtOH}$, rt, 4.5 h; b. 1.5 equiv. TBSOTf, 2 equiv. TEA, $\mathrm{CH}_{2} \mathrm{Cl}_{2}, \mathrm{rt}, 0.5$ h; c. 1.3 equiv. OsO THF, $-78{ }^{\circ} \mathrm{C}$ to rt, 20 h; d. 2 equiv. Dess-Martin, $\mathrm{CH}_{2} \mathrm{Cl}_{2}$, rt, 2 h; e. 0.25 equiv. TPAP, 3 equiv. NMO, Molecular Sieves, $\mathrm{CH}_{2} \mathrm{Cl}_{2}, \mathrm{rt}, 20 \mathrm{~h}$; f. 7.5 equiv. TBAF, THF, rt, 21 h; g. 2 equiv. $\mathrm{BF}_{3} \mathrm{OEt}_{2}, 6$ equiv. $\mathrm{Et}_{3} \mathrm{SiH}, \mathrm{CH}_{2} \mathrm{Cl}_{2}, 0{ }^{\circ} \mathrm{C}, 1.3$ h; h. 2.2 equiv. $\mathrm{Phl}(\mathrm{OAc})_{2}, 1$ equiv. $\mathrm{I}_{2}$, Cyclohexane/ $\mathrm{CH}_{2} \mathrm{Cl}_{2}(1: 1), 0{ }^{\circ} \mathrm{C}, 6 \mathrm{~h}$.

Scheme 4. Synthesis of $\mathrm{C} 12-\beta-\mathrm{OBz}, \mathrm{C} 17-\alpha-\mathrm{OH}$ rockogenin acetate. 
corresponding C16-oxo compound 22 was initially attempted with Dess-Martin periodinane. Instead of the desired C16-oxo compound 22, aldehyde $\mathbf{2 1}$ was obtained as a sole product ( $95 \%$ yield) in consequence of $\mathrm{C}-\mathrm{C}$ bond cleavage of spirobicyclic periodinane intermediate. ${ }^{13}$ To overcome this unwanted C-C bond cleavage between C16 and C17 position, the diol was subjected to TPAP (tetrapropylammonium perruthenate)-catalyzed oxidation condition to provide the desired C16-oxo compound 22 in 71\% yield over 2 steps (Scheme 4). ${ }^{14}$

Concomitant deprotection of the two silyl ether protecting groups at $\mathrm{C} 22$ and $\mathrm{C} 27$ with TBAF (tetrabutylammonium fluoride) provided hemiketal $\mathbf{2 3}$ quantitatively, which was further treated with 2 equiv. of $\mathrm{BF}_{3} \cdot \mathrm{OEt}_{2}$ and 6 equiv. $\mathrm{Et}_{3} \mathrm{SiH}$ to afford primary alcohol $\mathbf{2 4}$ in 55\% yield (Scheme 4). ${ }^{15}$

Alkoxyl radical is an electrophilic intermediate which is an efficient tool for hydrogen atom abstraction from nonactivated $\mathrm{C}-\mathrm{H}$ bonds. ${ }^{16}$ Especially, alkoxyl radical 1,5hydrogen transfer has been intensively used for spiroketalization in syntheses of many biologically active natural products. $^{17}$

In the final step, therefore, the primary alcohol $\mathbf{2 4}$ was subjected to Suarez alkoxyl radical cyclization condition to give $\mathrm{C} 12-\beta-\mathrm{OBz}, \mathrm{C} 17-\alpha-\mathrm{OH}$ rockogenin acetate 25 in $90 \%$ yield.

In conclusion, we have developed a novel and efficient method to smoothly open tetrahydrofuranyl rings of steroids to provide either cyclopentadienes or cyclopentenes depending upon the bond order between $\mathrm{C} 14$ and $\mathrm{C} 15$ positions, using freshly prepared TFAT (trifluoroacetyl trifluoromethanesulfonate) in the presence of an acid scavenger (2,6-ditert-butyl-4-methylpyridine).

Additionally, this method was applied to the synthesis of $\mathrm{C} 12-\beta-\mathrm{OBz}, \mathrm{C} 17-\alpha-\mathrm{OH}$ rockogenin acetate (12 steps from hecogenin acetate 1) where the $\mathrm{C} 17-\alpha-\mathrm{OH}$ group was successfully introduced without using 'Marker degradation'.

Thus, from the synthetic point of view, the above reaction affords an attractive synthesis to introduce a hydroxyl group to highly hindered $\mathrm{C} 17$ position without excision of the entire F-ring in the steroid sapogenin.

Acknowledgments. This work was partially supported by the KORDI (PE98641) and the Ministry of Land, Transport and Maritime Affairs (PM56641), Republic of Korea. We thank Daniel Jamieson (Department of Chemistry, Purdue University) for helping with the manuscript preparation.

\section{References}

1. Green, T. W.; Wuts, P. G. Protective Groups in Organic Synthesis, 4th ed.; John Wiley \& Sons, Inc.: Hoboken, New Jersy, 2007; Chapter 2.

2. (a) Forbus, T. R., Jr.; Taylor, S. L.; Martin, J. C. J. Org. Chem. 1987, 52, 4156. (b) Mandal, A. K.; Soni, N. R.; Ratnam, K. R. Synthesis 1985, 274. (c) Jung, M. E.; Lyster, M. A. J. Org. Chem.
1977, 42, 3761. (d) Olah, G. A.; Narang, S. C.; Balaram Gupta, B. G.; Malhotra, R. J. Org. Chem. 1979, 44, 1247. (e) Crombie, L.; Rainbow, L. J. J. Chem. Soc. Perkin Trans. 1 1994, 673. (f) Mudry, B.; Cohen, T. J. Am. Chem. Soc. 1991, 113, 1866. (g) Lee, J. S.; Fuchs, P. L. J. Am. Chem. Soc. 2005, 127, 13122. (h) Fortner, K. C.; Kato, D.; Tanaka, Y.; Shair, M. D. J. Am. Chem. Soc. 2010, 132, 275.

3. For representative instances, see: (a) Chinn, L. J. J. Org. Chem. 1967, 32, 687. (b) Shi, Y.; Jia, L.; Xiao, Q.; Lan, Q.; Tang, X.; Wang, D.; Li, M.; Ji, Y.; Zhou, T.; Tian, W. Chem. Asian J. 2011, 6, 786. (c) Izgu, E. C.; Burns, A. C.; Hoye, T. R. Org. Lett. 2011, 13, 703. (d) Welzel, P.; Janssen, B.; Duddeck, H. Liebigs Ann. Chem. 1981, 546. (e) Iglesias-Arteaga, M. A.; Arcos-Ramos, R. O. Tetrahedron Lett. 2006, 47, 8029. (f) Lee, S.; Jamieson, D.; Fuchs, P. L. Org. Lett. 2009, 11, 5. (g) Jiang, B.; Shi, H.-P.; Xu, M.; Wang, W.-J.; Zhou, W.-S. Tetrahedron 2008, 64, 9738. (h) Izzo, I.; Di Filippo, M.; Napolitano, R.; De Riccardis, F. Eur. J. Org. Chem. 1999, 3505. (i) Chen, L.-J.; Xu, Q.-H.; Huang, H.; Lin, J.-R.; Tian, W.-S. Tetrahedron Lett. 2007, 48, 3475. (j) Lee, J. S.; Fuchs, P. L. Org. Lett. 2003, 5, 3619.

4. (a) Marker, R. E.; Rohrmann, E. J. Am. Chem. Soc. 1939, 61, 846. (b) Marker, R. E.; Krueger, J. J. Am. Chem. Soc. 1940, 62, 3349. (c) Marker, R. E. J. Am. Chem. Soc. 1940, 62, 2543. (d) Woodward, R. B.; Sondheimer, F.; Taub, D. J. Am. Chem. Soc. 1951, 73, 4057.

5. (a) Jeong, J. U.; Sutton, S. C.; Kim, S.; Fuchs, P. L. J. Am. Chem. Soc. 1995, 117, 10157. (b) Jeong, J. U.; Guo, C.; Fuchs, P. L. J. Am. Chem. Soc. 1999, 121, 2071. (c) LaCour, T. G.; Guo, C.; Bhandaru, S.; Boyd, M. R.; Fuchs, P. L. J. Am. Chem. Soc. 1998, 120,692 .

6. Lee, J. S. Encyclopedia of Reagents for Organic Synthesis, 2nd ed.; Paquette, L. A., Crich, D., Fuchs, P. L., Molander, G. A., Eds.; John Wiley \& Sons, Ltd.: Chichester, West Sussex, 2009; Vol. 12, p 9989.

7. Even TBS, a more acid-sensitive protection group tolerated in the same reaction condition to provide the corresponding product, see: Lee, J. S. Ph.D. Dissertation, Purdue University, West Lafayette, IN, 2005.

8. Lee, S.; Fuchs, P. L. Org. Lett. 2004, 6, 1437.

9. Velgova, H.; Kasal, A.; Budesinsky, M. Steroids 1994, 59, 335.

10. Each compound was separated by HPLC.

11. Toro, A.; Pallagi, I.; Ambrus, G. Tetrahedron Lett. 1994, 35, 7651.

12. Kim, M.; Kawada, K.; Gross, R. S.; Watt, D. S. J. Org. Chem. 1990, 55,504

13. De Munari, S.; Frigerio, M.; Santagostino, M. J. Org. Chem. 1996, 61, 9272

14. Lenz, R.; Ley, S. V. J. Chem. Soc., Perkin Trans. 1997, 1, 3291.

15. Lee, J. S. Ph.D. Dissertation, Purdue University, West Lafayette, IN, 2005.

16. For a review, see: (a) Cekovic, Z. Tetrahedron 2003, 59, 8073. (b) Feray, L.; Kuznetsov, N.; Renaud, P. In Radicals in Organic Synthesis; Renaud, P., Sibi, M. P., Eds.; Wiley-VCH: Weinheim, Germany, 2001; Vol. 2, p 246.

17. (a) Ravindar, K.; Reddy, M. S.; Lindqvist, L.; Pelletier, J.; Deslongchamps, P. J. Org. Chem. 2011, 76, 1269. (b) Lee, J. S.; Fuchs, P. L. Org. Lett. 2003, 5, 2247. (c) Betancor, C.; Freire, R.; Perez-Martin, I.; Prange, T.; Suarez, E. Org. Lett. 2002, 4, 1295. (d) Lee, S.; LaCour, T. G.; Lantrip, D.; Fuchs, P. L. Org. Lett. 2002, 4, 313. (e) Lee, S.; Fuchs, P. L. Org. Lett. 2002, 4, 317. (f) Barrero, A. F.; Oltra, J. E.; Alvarez, M.; Rosales, A. J. Org. Chem. 2002, 67, 5461. (g) Barrero, A. F.; Oltra, J. E.; Alvarez, M. Tetrahedron Lett. 2000, 41, 7639. (h) Chatgilialoglu, C.; Gimisis, T.; Spada, G. P. Chem.-Eur. J. 1999, 2866. (i) Allen, P. A.; Brimble, M. A.; Prabaharan, H. Synlett 1999, 295. (j) Petrovic, G.; Saicic, R. N.; Cekovic, Z. Synlett 1999, 635. 